\title{
Correction of humpback and DISI deformities by vascularized bone grafting in patients with scaphoid nonunion
}

\author{
Nana Nagura $^{1}$, Kiyohito Naito ${ }^{1, *}$, Yoichi Sugiyama ${ }^{1,3}$, Hiroyuki Obata ${ }^{1,2}$, Kenji Goto ${ }^{1}$, Ayaka Kaneko ${ }^{1,2}$, \\ Yoshimasa Tomita ${ }^{4}$, Yoshiyuki Iwase ${ }^{3}$, Kazuo Kaneko ${ }^{1}$, and Muneaki Ishijima ${ }^{1,2}$ \\ ${ }^{1}$ Department of Orthopaedics, Juntendo University Faculty of Medicine, 2-1-1 Hongo, Bunkyo-ku, 113-8421 Tokyo, Japan \\ 2 Department of Medicine for Orthopaedics and Motor Organ, Juntendo University Graduate School of Medicine, 2-1-1 Hongo, \\ Bunkyo-ku, 113-8421 Tokyo, Japan \\ 3 Department of Orthopeadic Surgery, Juntendo Tokyo Koto Geriatric Medical Center, 3-3-20 Shisuna, Koto-ku, 136-0075 Tokyo, Japan \\ ${ }^{4}$ Department of Orthopaedic Surgery, Japan Labour Health and Safety Organization Tokyo Rosai Hospital, 4-13-21 Omori-Minami, \\ Ota-ku, 143-0013 Tokyo, Japan
}

Received 9 October 2020, Accepted 20 February 2021, Published online 11 March 2021

\begin{abstract}
Introduction: Although vascularized bone grafting (VBG) using 1, 2 intercompartmental supraretinacular artery (1, 2 ICSRA) is effective for scaphoid nonunion, dorsal intercalated segment instability (DISI) deformity persists even after correction of humpback deformity (HD). The purpose of this retrospective study was to evaluate the correction of HD and DISI deformity after 1, 2 ICSRA VBG for scaphoid nonunion. Methods: We treated 18 patients (mean age: 25.8, 16 males and 2 females) with scaphoid nonunion using a 1, 2-ICSRA VBG between January 2010 and December 2018. The average time from injury to surgery was 20.0 (3-120) months. The nonunions were located at the waist in all patients. The correction of HD and DISI deformity was investigated on the preoperative images and images at the last examination. Results: In all patients, the correction of HD was positively correlated with that of DISI deformity. Moreover, we focused on the time from injury to surgery and evaluated changes in HD and DISI deformity according to the time to surgery. As a result, changes in HD and DISI deformity were positively correlated in patients with a shorter time to surgery but were not correlated when the time to surgery exceeded 5 months. Conclusions: These results suggest that DISI deformity can be corrected by correcting HD when the time from injury to surgery is short, but that correction is difficult if the time to surgery is prolonged.
\end{abstract}

Key words: Scaphoid nonunion, Humpback deformity, Dorsal intercalated segment instability deformity, The time to surgery, 1, 2 intercompartmental supraretinacular artery.

\section{Introduction}

Vascularized pedicled radial grafting for scaphoid nonunion reported in 1991 by Zaidemberg ( $\mathrm{Z}$ procedure) is autologous bone grafting using the ascending irrigating branch of the radius, i.e., 1, 2 intercompartmental supraretinacular artery (1, 2 ICSRA) as the feeding vessel [1]. Vascularized bone grafting (VBG) is effective for the treatment of scaphoid nonunion, particularly, in refractory cases, such as those accompanied by avascular necrosis (AVN), those with a history of surgery, those with a long period after injury, and those of the proximal third of the scaphoid [2]. The outcomes of the $\mathrm{Z}$ procedure are satisfactory in refractory cases $[3,4]$.

On the other hand, Ribak et al. compared the outcomes of VBG and non-VBG for scaphoid nonunion including nonunion in the waist. As a result, it was reported that VBG had a higher

\footnotetext{
*Corresponding author: knaito@juntendo.ac.jp
}

bone union rate and a shorter time to a bone union than nonVBG [5]. From this evidence, we use VBG to treat scaphoid nonunions in cases of poor blood flow in proximal bone fragments and cases in which the time from injury to surgery is long, even for nonunions in the waist.

One of the factors for dorsal intercalated segment instability (DISI) deformity associated with scaphoid nonunion is humpback deformity (HD) due to bone defect on the volar side of the fracture site [6]. Moreover, the proximal fragment flexes dorsally with the lunate bone to cause DISI deformity [7, 8], and persistence of DISI deformity is reported to be a factor of poor clinical prognosis $[9,10]$. Therefore, correction of HD and DISI deformity is important along with bone union for improving the clinical outcome of nonunion and preventing scaphoid nonunion advanced collapse (SNAC) wrist. However, the surgical technique in the treatment of scaphoid nonunion is still controversial. Kim et al. reported autologous iliac bone grafts for scaphoid nonunion showed a significant correlation 
between correction of HD and correction of DISI deformity [9]. On the other hand, in the $\mathrm{Z}$ procedure, where the amount of bone graft is limited, the reduction that can be obtained after surgery remains questionable [11]. Moreover, there is no literature describing the correlation between correction of both deformities after VBG for scaphoid nonunion. Therefore, the correction of HD and DISI deformity after VBG for scaphoid nonunion were evaluated, then the relationship between the degree of correction of HD and DISI deformity and the time to surgery was evaluated in this study.

\section{Materials and methods}

\section{Patients}

This study was approved by the ethics committee for medical research of our university (No. 18-323), and informed consent was received from all patients and the patients of minor patients under the age of 20.

Between January 2010 and December 2018, 1, 2 ICSRA VBG using was performed for scaphoid nonunion in 18 patients with a mean age of 25.8 (16-46) years consisting of 16 males and 2 females. The average time from injury to surgery was $20.0(3-120)$ months. The fracture type at the time of injury by the Herbert classification was B1 in 6 and B2 in 12, and the nonunions were located at the waist in all patients.

\section{Surgical technique}

Surgery was performed under general anesthesia. According to the report by Zaidemberg et al., the 1, 2 ICSRA running dorsally between the first and second compartments of the extensor tendon was identified, a graft including this vessel was raised from the radius and placed at the site of nonunion [1]. Curettage of the site of nonunion was performed from the volar side in patients with relatively large HD but from the dorsal side in the others, and the size of the graft to be placed was measured. In patients judged by preoperative imaging to have relatively severe DISI deformity, the radius and lunate bone were temporarily fixed for intraoperative correction of DISI deformity by inserting a Kirschner wire from the dorsal side of the radius toward the lunate bone while correcting the radiolunate (RL) angle to $0^{\circ}$ by volarly flexing the wrist, and the graft was placed in this condition. Headless compression screws were used for the fixation of the nonunion. After surgery, thumb spica splinting was applied for 4 weeks, and range of motion exercise was initiated thereafter.

\section{Imaging assessment}

To evaluate the degree of correction of HD and DISI deformity by 1, 2 ICSRA VBG for scaphoid nonunion, the preoperative images and images at the last examination by plain radiography and computed tomography (CT) were compared. At our hospital, preoperative CT was used to evaluate the condition of the nonunion site and scaphoid alignment, and postoperative CT was used to evaluate bone union and scaphoid

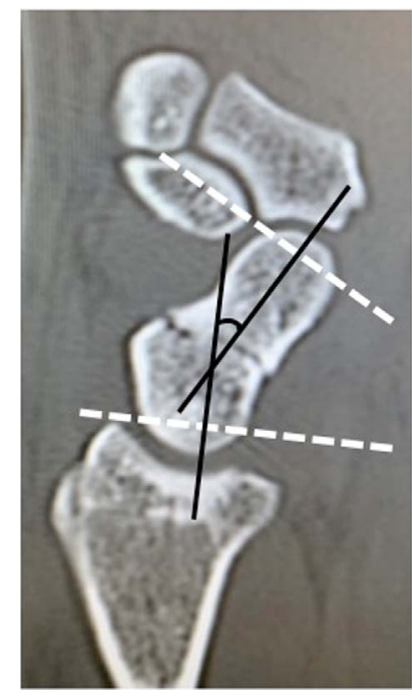

(A)

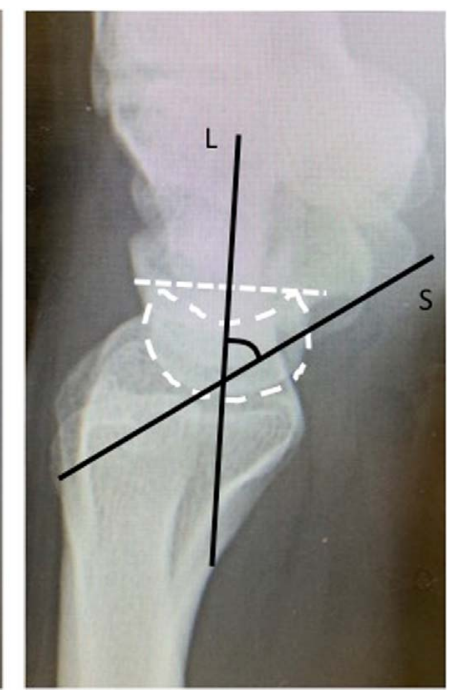

(B)
Figure 1. Method for the measurement of the intrascaphoid angle (ISA) (A) and scapholunate angle (SLA) (B). (A) ISA was measured as the angle between the perpendicular lines on the distal and proximal articular surfaces of the scaphoid in the lateral CT image. (B) SLA was measured as the angle between the long axis of the scaphoid (tangent on the volar cortex of the scaphoid) and the long axis of the lunate (axis connecting the midpoints of the proximal and distal arcs) in the lateral view of plain radiography of the wrist.

alignment in all patients. The intrascaphoid angle (ISA) was examined as an index of $\mathrm{HD}$, and the scapholunate angle (SLA) was examined as an index of DISI deformity. ISA is an angle formed by two lines that are perpendicular to the proximal and distal articular surfaces in the lateral view on CT (Figure 1A), and SLA is the angle between the long axis of the scaphoid (tangent line of the volar cortex of the scaphoid) and the long axis of the lunate bone (the line between the midpoints of the proximal and distal arcs) in the lateral view of plain radiography of the wrist (Figure 1B). Measurements of ISA and SLA were performed by an independent blind orthopaedic surgeon.

\section{Evaluations}

In this study, to assess the improvement in HD and DISI deformity, ISA and SLA were compared preoperatively and at the time of the last postoperative examination. In addition, the relationship between the degree of correction of $\mathrm{HD}$ and DISI deformity and the time to surgery was evaluated according to the time after injury ( $<4$ months vs. $\geq 4$ months, $<5$ months vs. $\geq 5$ months, and $<6$ months vs. $\geq 6$ months).

\section{Statistical analysis}

The data obtained by imaging examinations were compared using the Mann-Whitney $U$-test. The correlation of the changes in the data between before and after surgery was evaluated by calculating Spearman's correlation coefficient. The level of significance was $p<0.05$. 
Table 1. Period from injury to surgery and changes in the intrascaphoid angle (ISA) and scapholunate angle (SLA) between before and after surgery.

\begin{tabular}{|c|c|c|c|c|c|c|}
\hline & \multicolumn{3}{|c|}{ ISA } & \multicolumn{3}{|c|}{ SLA } \\
\hline & Before & After & Change & Before & After & Change \\
\hline All patients $(n=18)$ & $37.8 \pm 18.5$ & $20.2 * \pm 12.9$ & $17.5 \pm 14.9$ & $63.9 \pm 12.7$ & $52.6 \pm 18.6$ & $8.9 \pm 11.2$ \\
\hline Time to surgery $<4$ months $(n=5)$ & $36.4 \pm 22.6$ & $18.6 \pm 11.5$ & $17.8 \pm 16.0$ & $60.2 \pm 15.3$ & $52.6 \pm 13.3$ & $7.6 \pm 11.8$ \\
\hline Time to surgery $\geq 4$ months $(n=13)$ & $38.4 \pm 17.7$ & $20.9 * \pm 13.8$ & $17.5 \pm 15.1$ & $65.3 \pm 11.9$ & $52.7 \pm 20.7$ & $9.4 \pm 11.4$ \\
\hline Time to surgery $<5$ months $(n=9)$ & $41.9 \pm 18.3$ & $23.0^{*} \pm 14.1$ & $18.9 \pm 17.5$ & $61.6 \pm 13.4$ & $56.0 \pm 14.4$ & $6.9 \pm 11.5$ \\
\hline Time to surgery $\geq 5$ months $(n=9)$ & $33.7 \pm 18.9$ & $17.6^{*} \pm 11.7$ & $16.2 \pm 12.7$ & $66.2 \pm 12.3$ & $49.6 \pm 22.1$ & $10.9 \pm 11.2$ \\
\hline Time to surgery $<6$ months $(n=11)$ & $41.9 \pm 19.1$ & $20.5^{*} \pm 14.5$ & $21.4 \pm 16.8$ & $65.2 \pm 15.0$ & $55.9 \pm 14.2$ & $10.5 \pm 12.4$ \\
\hline Time to surgery $\geq 6$ months $(n=7)$ & $34.3 \pm 18.4$ & $19.7 \pm 12.4$ & $14.6 \pm 10.7$ & $63.7 \pm 9.7$ & $55.6 \pm 16.0$ & $7.9 \pm 10.3$ \\
\hline
\end{tabular}

Statistical analysis of the difference between before and after surgery:

$* p<0.05$.ISA: Intrascaphoid angle, SLA: Scapholunate angle.

Table 2. Correlations between the intrascaphoid angle (ISA) and scapholunate angle (SLA).

\begin{tabular}{lc}
\hline & All patients $(n=18)$ \\
& $0.54^{*}$ \\
\hline Time to surgery $<4$ months $(n=5)$ & Time to surgery $\geq 4$ months $(n=13)$ \\
$r=0.78^{*}$ & $r=0.42^{*}$ \\
Time to surgery $<5$ months $(n=9)$ & Time to surgery $\geq 5$ months $(n=9)$ \\
$r=0.89^{*}$ & $r=0.18$ \\
Time to surgery $<6$ months $(n=11)$ & Time to surgery $\geq 6$ months $(n=7)$ \\
$r=0.79^{*}$ & $r=-0.11$ \\
\hline
\end{tabular}

Statistical analysis of the correlation between ISA and SLA:

${ }^{*} p<0.05$ and $\left|r_{s}\right|>0.4$.ISA: Intrascaphoid angle, SLA: Scapholunate angle.

\section{Results}

\section{Measurements of ISA and SLA, and the correlation between ISA and SLA in all patients}

The mean postoperative follow-up period was $15.7 \pm 2.5$ months. Of the 18 patients, nonunion was healed in 15 and not in 3 , with a union rate of $83.3 \%$. The mean time to union was 4.0 (2-7) months. ISA determined by CT was $37.8 \pm 18.5^{\circ}$ preoperatively and was significantly improved to $20.2 \pm 12.9^{\circ}$ at the time of last postoperative examination $(p<0.05)$. SLA determined by plain radiography was $63.9 \pm 12.7^{\circ}$ preoperatively and improved to $52.6 \pm 18.6^{\circ}$ at the time of the last examination $(p=0.06)$ (Table 1). The changes in ISA and SLA at the time of the last examination compared with the preoperative values were $17.5 \pm 14.9$ and $8.9 \pm 11.2$, respectively, and showed a positive correlation $(r=0.54, p<0.05)$ (Table 2).

\section{Comparison according to the time to surgery (<4 months vs. $\geq 4$ months)}

In the 5 patients ( 5 males and 0 females) in whom the time to surgery was $<4$ months, ISA was $36.4 \pm 22.6^{\circ}$ preoperatively and improved to $18.6 \pm 11.5^{\circ}$ at the time of the last examination $(p=0.13)$, and SLA improved from $60.2 \pm 15.3^{\circ}$ before surgery to $52.6 \pm 13.3^{\circ}$ at the time of the last examination ( $p=0.33$ ) (Table 1$)$. In the 13 patients ( 11 males and 2 females) in whom the time to surgery was $\geq 4$ months, ISA significantly improved from $38.4 \pm 17.7^{\circ}$ before surgery to $20.9 \pm 13.8^{\circ}$ at the time of the last examination $(p<0.05)$, and SLA improved from $65.3 \pm 11.9^{\circ}$ before surgery to $52.7 \pm 20.7^{\circ}$ at the time of the last examination $(p=0.09)$. The change in ISA from before surgery to the time at the last examination was $17.8 \pm 16.0$ in the $<4$ months group and $17.5 \pm 15.1$ in the $\geq 4$ months group and the change in SLA was $7.6 \pm 11.8$ and $9.4 \pm 11.4$, respectively. The changes in ISA and SLA were correlated in both groups $(r=0.78, p<0.05 ; r=0.42, p<0.05)$ (Table 2 ).

\section{Comparison according to the time to surgery (<5 months vs. $\geq 5$ months)}

In the 9 patients ( 9 males and 0 females) in whom the time to surgery was $<5$ months, ISA significantly improved from $41.9 \pm 18.3^{\circ}$ before surgery to $23.0 \pm 14.1^{\circ}$ at the time of the last examination $(p<0.05)$, and SLA improved from $61.6 \pm 13.4^{\circ}$ to $56.0 \pm 14.4^{\circ}(p=0.31)$ (Table 1$)$. In the 9 patients in whom the time to surgery was $\geq 5$ months ( 7 males and 2 females), ISA improved significantly from $33.7 \pm 18.9^{\circ}$ before surgery to $17.6 \pm 11.7^{\circ}$ at the time of the last examination $(p<0.05)$, and SLA improved from $66.2 \pm 12.3^{\circ}$ to $49.6 \pm 22.1^{\circ}(p=0.11)$. The change in ISA from before surgery to the time of the last examination was $18.9 \pm 17.5$ in the $<5$ months group and $16.2 \pm 12.7$ in the $\geq 5$ months group and the change in SLA was $6.9 \pm 11.5$ and $10.9 \pm 11.2$, respectively. The changes in ISA and SLA were positively correlated $(r=0.89, p<0.05)$ in the $<5$ months group but not in the $\geq 5$ months group $(r=0.18, p>0.05)$ (Table 2). 


\section{Comparison according to the time to surgery ( $<6$ months vs. $\geq 6$ months)}

In the 11 patients (10 males and 1 female) in whom the time to surgery was $<6$ months, ISA improved from $41.9 \pm 19.1^{\circ}$ before surgery to $20.5 \pm 14.5^{\circ}$ at the time of the last examination $(p=0.06)$, and SLA improved from $65.2 \pm 15.0^{\circ}$ to $55.9 \pm 14.2^{\circ}(p=0.13)$ (Table 1$)$. In the 7 patients $(6$ males and 1 female) in whom the time to surgery was $\geq 6$ months, ISA significantly improved from $34.3 \pm 18.4^{\circ}$ before surgery to $19.7 \pm 12.4^{\circ}$ at the time of the last examination $(p=0.06)$, and SLA improved from $63.7 \pm 9.7^{\circ}$ to $55.6 \pm 16.0^{\circ}$ $(p=0.27)$. The change in ISA from before surgery to the time of the last examination was $21.4 \pm 16.8$ in the $<6$ months group and $14.6 \pm 10.7$ in the $\geq 6$ months group and the change in SLA was $10.5 \pm 12.4$ and $7.9 \pm 10.3$, respectively. The changes in ISA and SLA were positively correlated in the $<6$ months group $(r=0.79, p<0.05)$ but not correlated in the $\geq 6$ months group $(r=-0.11, p>0.05)$ (Table 2).

\section{Discussion}

The size of bone grafts in the treatment of scaphoid nonunion is still controversial. Generally, to correct HD associated with scaphoid nonunion, it is necessary to graft a sufficient amount of bone as a support on the volar side [11]. Among the VBGs, the one placed from the volar side of the radius proposed by Judet et al. has been reported to have allowed the collection of larger grafts and resulted in a satisfactory bone union rate compared with the $\mathrm{Z}$ procedure, by which grafts are collected from the dorsal side of the radius $[12,13]$. In addition, free VBG from the medial condyle of the femur allows the collection of larger grafts compared with other pedicled VBGs, and favorable clinical results have been reported [14]. The $\mathrm{Z}$ procedure is inferior to these techniques in the graft size $[11,15]$. However, as observed above, for the treatment of refractory scaphoid nonunion, the outcomes of the $\mathrm{Z}$ procedure have been reported to be generally favorable, and the technique is a useful option of surgical treatment $[3,4,13]$.

There are several limitations in this study. The first is a method for evaluation of the alignment of the scaphoid. Recently, several studies using the scaphoid length as an indicator of reduction and carpal alignment were reported $[16,17]$. Moreover, Kim et al. reported that the shortening of the scaphoid caused DISI deformity [9]. However, the scaphoid length has a gender difference and laterality $[18,19]$, and it can be difficult to use it as an evaluation for the reduction of the scaphoid nonunion. Therefore, in the cases of this study, when the $\mathrm{Z}$ procedure was performed from the volar side, the continuity of the dorsal site was maintained, and bone grafting was performed on the wedge-shaped bone defect to reduce the alignment. In addition, when the $\mathrm{Z}$ procedure was performed from the dorsal side, the continuity of the volar site was also maintained. Based on these findings, although the shortening of the scaphoid itself has not been strictly evaluated, ISA was used as the evaluation of HD in this study. Therefore, it cannot be asserted that the cause of residual DISI deformity in this study is only the involvement of subclinical damage to the soft tissues such as the SL ligament. Next, the indication of the $\mathrm{Z}$ procedure is still controversial. In previous reports, Chang et al. reported that the $\mathrm{Z}$ procedure was performed on nonunions with $\mathrm{HD}$ and $\mathrm{AVN}$, but the bone union was not obtained and good treatment results could not be obtained [15]. In addition, Derby et al. reported that VBG was relatively contraindicated in cases with HD and carpal instability [20]. However, in Japan, Kawasaki et al. reported favorable outcomes of the $\mathrm{Z}$ procedure and the correction of DISI for scaphoid nonunion with HD [21]. And there is a current situation among Japanese hand surgeons that, contrary to reports in previous English literature, the $\mathrm{Z}$ procedure is preferred for this nonunion. In fact, we have also found improvement in $\mathrm{HD}$ using the $\mathrm{Z}$ procedure for cases with $\mathrm{HD}$, and we think that the $\mathrm{Z}$ procedure may be useful for cases with a relatively short time to injury.

Indeed, in our patients, ISA improved significantly at the time of the last examination compared with before surgery, indicating that the $\mathrm{Z}$ procedure allows the collection of a sufficient amount of bone for correction of HD (Table 1). In addition, the change in ISA at the time of the last examination compared with the value before surgery was positively correlated with the change in SLA, indicating that DISI deformity was improved by correcting HD (Table 2). However, in some of the 18 patients evaluated in this study, correction of DISI deformity was insufficient even when HD could be corrected.

Concerning correction of DISI associated with scaphoid nonunion, Fisk et al. reported in a study about wedge grafts that the volar radiocarpal ligament tension could be normalized, and DISI could be corrected, by the restoration of the length of the scaphoid bone shortened by HD [6]. Although the SL ligament is the primary ligament that contributes to scaphoid stability, the radioscaphocapitate (RSC) ligament and long RL ligament, which are volar radiocarpal ligaments, are reported to contribute secondarily to stability [22]. Therefore, we directed our attention to the ligaments around the scaphoid bone as a factor of insufficient correction of DISI despite correction of HD. We hypothesized that the history of HD is prolonged in longstanding scaphoid nonunion and that correction of DISI deformity is made difficult by changes in the length and elasticity of the surrounding ligaments despite the possibility of bony correction of HD. In this study, we focused on the period from injury to surgery and evaluated changes in HD and DISI deformity according to the time to surgery $(<4,5$, and 6 months vs. $\geq 4,5$, and 6 months). As a result, changes in HD and DISI deformity were positively correlated in patients with a shorter time to surgery but were not correlated when the time to surgery exceeded 5 months (Table 2). These results suggest that DISI deformity can be corrected by correcting HD when the time from injury to surgery is short but that correction of DISI deformity by correction of HD is difficult if the time to surgery is prolonged. Thus, in patients with scaphoid nonunion, HD may be corrected, but DISI may not be sufficiently corrected, by the $\mathrm{Z}$ procedure if the time from injury to surgery is long.

Capito and Higgins prepared models mimicking scaphoid nonunion accompanied by DISI deformity using cadavers by cutting the SL ligament, which stabilizes the scaphoid bone, RSC ligament, and long RL ligament [22]. They showed that 
DISI deformity can be corrected without restoration of the ligaments by inserting a bone graft slightly larger than the actual bone defect [22]. Our present study suggested that the $\mathrm{Z}$ procedure is a useful option for surgical treatment of scaphoid nonunion but that its effectiveness for the correction of DISI deformity is questionable if surgery is performed more than 5 months after injury, and the effect of intracarpal ligaments is considered a factor of such an outcome.

\section{Declarations}

\section{Funding} study.

The authors declare that no funding was involved in this

\section{Conflict of interest}

The authors declare that they have no conflict of interest.

\section{Ethics approval}

The study was approved by the ethics committee for medical research of our university (No. 18-323).

\section{Consent to participate}

Informed consent was received from all patients.

\section{Consent for publication}

Informed consent was received from all patients.

\section{Availability of data and material}

The datasets during and/or analysed during the current study available from the corresponding author on reasonable request.

\section{Code availability}

The datasets during and/or analysed during the current study available from the corresponding author on reasonable request.

\section{Authors' contributions}

NN (first author) mainly wrote this manuscript, acquisition of data, analysis and interpretation of data. $\mathrm{KN}$ (corresponding author), YS, YI, KK and MI mainly performed conception and design of this study. $\mathrm{HO}, \mathrm{KG}, \mathrm{AK}$, and YT performed acquisition of data, analysis and interpretation of data.

\section{References}

1. Zaidemberg C, Siebert JW, Angrigiani C (1991) A new vascularized bone graft for scaphoid nonunion. J Hand Surg Am 16, 474-478.
2. Merrell GA, Wolfe SW, Slade JF 3rd (2002) Treatment of scaphoid nonunions: Quantitateive meta-analysis of the literature. J Hand Surg Am 27, 685-691.

3. Steinmann SP, Bishop AT, Berger RA (2002) Use of the 1, 2 intercompartmental supraretinacular artery as a vascularized pedicle bone graft for difficult scaphoid nonunion. J Hand Surg Am 27, 391-401.

4. Waters PM, Stewart SL (2002) Surgical treatment of nonunion and avascular necrosis of the proximal part of the scaphoid in adolescents. J Bone Joint Surg Am 84, 915-920.

5. Ribak S, Medina CE, Mattar R Jr, Ulson HJ, Ulson HJ, Etchebehere M (2010) Treatment of scaphoid nonunion with vascularised and non-vascularised dorsal bone grafting from the distal radius. Int Orthop 34, 683-688.

6. Fisk GR (1970) Carpal instability and the fractured scaphoid. Ann R Coll Surg Engl 46, 63-76.

7. Moritomo H, Murase T, Oka K, Tanaka H, Yoshikawa H, Sugamoto K (2008) Relationship between the fracture location and the kinematic pattern in scaphoid nonunion. J Hand Surg Am 33, 1459-1468.

8. Oka K, Murase T, Moritomo H, Goto A, Sugamoto K, Yoshikawa H (2005) Patterns of bone defect in scaphoid nonunion: A 3-dimensional and quantitative analysis. J Hand Surg Am 30, 359-365.

9. Kim JH, Lee KH, Lee BG, Lee CH, Kim SJ, Choi WS (2017) Dorsal intercalated segmental instability associated with malunion of a reconstructed scaphoid. J Hand Surg Eur 42, 240-245.

10. Tsuyuguchi Y, Murase T, Hidaka N, Ohno H, Kawai H (1995) Anterior wedge-shaped bone graft for old scaphoid fractures or non-unions. J Hand Surg Br 20, 194-200.

11. Jones DB, Burger H, Bishop AT, Shin AY (2008) Treatment of scaphoid waist nonunions with an avascular proximal pole and carpal collapse. A comparison of two vascularized bone grafts. J Bone Joint Surg Am 90, 2616-2625.

12. Judet R, Roy-Camille R, Guillamon JL (1972) Treatment of nonunion of the carpal scaphoid by pediculated graft. Rev Chir Orthop Reparatrice Appar Mot 58, 699-705.

13. Mallizos KN, Dailiana Z, Varitimidis S, Koutalos A (2017) Management of scaphoid nonunions with vascularized bone grafts from the distal radius: Mid- to long- term follow-up. Eur J Orthop Surg Traumatol 27, 33-39.

14. Doi K, Oda T, Soo-Heong Nanda V (2002) Free vascularized bone graft for nonunion of the scaphoid. J Hand Surg Am 25, 507-519.

15. Chang MA, Bishop AT, Moran SL, Shin AY (2006) The outcomes and complications of 1, 2-intercompartmental supraretinacular artery pedicled vascularized bone grafting of scaphoid nonunions. J Hand Surg Am 31, 387-396.

16. Mathoulin CL, Arianni M (2018) Treatment of the scaphoid humpback deformity - Is correction of the dorsal intercalated segment instability deformity critical? J Hand Surg Eur 43, $13-23$.

17. Elatta MA, Elglaind SM, Talat E, Alqaseer AM, Basheer HM (2019) Scapho-capitate ratio for estimation of scaphoid length. J Hand Surg Asian Pac 24, 202-207.

18. Heinzelmann AD, Archer G, Bindra RR (2007) Anthropometry of the human scaphoid. J Hand Surg Am 32, 1005-1008.

19. Smith DK (1993) Anatomic features of the carpal scaphoid: Validation of biometric measurements and symmetry with threedimensional MR imaging. Radiology 187, 187-191. 
20. Derby BM, Murray PM, Shin AY, Bueno RA, Mathoulin CL, Ade T, Neumeister MW (2013) Vascularized bone grafts for the treatment of carpal bone pathology. Hand (N Y) 8, 27-40.

21. Kawasaki K, Nemoto T, Kubo K, Yomita K, Inagaki K (2020) Vascularized bone grafting for scaphoid nonunion with
Humpback deformity: The surgical technique. J Wrist Surg 9, 528-534.

22. Capito AE, Higgins JP (2013) Scaphoid overstuffing: The effects of the dimensions of scaphoid reconstruction on scapholunate alignment. J Hand Surg Am 38, 2419-2425.

Cite this article as: Nagura N, Naito K, Sugiyama Y, Obata H, Goto K, Kaneko A, Tomita Y, Iwase Y, Kaneko K \& Ishijima M (2021) Correction of humpback and DISI deformities by vascularized bone grafting in patients with scaphoid nonunion. SICOT-J 7, 13 